How to reference this article

Bal, E. (2019). Contro la minaccia della cultura locale. Gli aspetti performativi dell'identità culturale in mPalermu e ne La Carnezzeria di Emma Dante. Italica Wratislaviensia, 10(2), 275-288.

DOI: http://dx.doi.org/10.15804/IW.2019.10.1.29

Ewa Bal

Uniwersytet Jagielloński w Krakowie

ewa.bal@uj.edu.pl

ORCID: 0000-0002-2434-6108

\title{
CONTRO LA MINACCIA DELLA CULTURA LOCALE. GLI ASPETTI PERFORMATIVI DELL'IDENTITÀ CULTURALE IN MPALERMU E NE LA CARNEZZERIA DI EMMA DANTE
}

\author{
AGAINST THE MENACE OF THE LOCAL CULTURE. \\ PERFORMATIVE ASPECTS OF CULTURAL IDENTITY \\ IN MPALERMU AND CARNEZZERIA BY EMMA DANTE
}

\begin{abstract}
Taking into account important changes that have taken place in the last 20 years in the fields of performance studies and cultural studies (especially de- and post-colonial studies) regarding the way in which local cultures and ethnicity are represented in the theatre and interpreted in the humanities, this article revises the ways of presenting local cultures in the Italian dialect theatre of the last decade. This analysis shows how a hegemonic (at least until recently) dramatic model developed by Eduardo de Filippo and based on producing nostalgia towards an idealised past of a local culture needs to be critically reformulated, especially from the perspective of a young generation of female artists who work in the field of dialect theatre.

To this end, in this article, the author analyses the performing strategies of two of Emma Dante's plays, mPalermu and Carnezzeria, to prove that localness is not an essentially understood characteristic of a certain community, but, rather, it should be seen as a complex performative, repetitive practice that is both bodily and discursive. Conversely, the author points out that these practices should be examined in depth and seen as a kind of cultural oppression that serves as a camouflage of some deeper imprinted relationships based on violence and domination. The examples of Emma Dante's plays from the beginning of her career allow the author to also cast a critical gaze at an issue of primordialism, raised by Arjun Appadurai and seen as a way of viewing the Other. The latter reflects a voyeuristic pleasure of the Western world, coming from the sense of dominance over allegedly underdeveloped and primitive cultures. Emma Dante's theatre, according to the author of this article, is an example of de-colonial artistic practice, as it subversively uses the voyeuristic inclinations of the viewer to denounce the violent character of local Sicilian scripts of behaviour that, until now, had usually been left unsaid in public debate.
\end{abstract}

Keywords: localness, cultural identity, dialect theatre, Emma Dante, performativity 
Tegli ultimi venticinque anni, gli studi sulle performance delle cul1 ture locali ed etniche e sugli aspetti performativi delle identità culturali (Appadurai, 1995; Clifford, 1996; Bhaba, 2010; Butler, 1993, 2002 e 2003) si sono allontanati dall'atteggiamento scientifico essenzialistico che considerava le pratiche culturali delle determinate comunità locali come espressione di una cultura indigena effettivamente esistente e legata ad un determinato territorio. Si è diffuso invece un approccio diverso, molto più critico e distanziato, che si può riassumere in due filoni. Da una parte la nozione del locale, per lo meno nel teatro, viene osservata e studiata dal punto di vista delle strategie performative attraverso le quali gli artisti teatrali stabiliscono il rapporto, spesso conflittuale o critico, con un determinato territorio e comunità (Carlson, 2001 e 2006). Dall'altra però, grazie agli studi post e de-coloniali si è cominciato ad indagare come un determinato tipo di teatro dialettale viene posizionato o situato in un contesto discorsivo e culturale più vasto (per esempio nel contesto storico del teatro nazionale o europeo) da parte degli storici e dei critici del teatro ${ }^{1}$. Gli studiosi si sono chiesti soprattutto come si possa revisionare la versione canonica della storia nazionale del teatro, cambiando o sovvertendo il rapporto tra fenomeni situati finora nel suo centro e nelle periferie, oppure riscrivendola dalla prospettiva dei gender, queer e gay studies. In Italia questo complicato e dialettico rapporto del teatro dialettale con il proprio contesto culturale nonché il suo posizionamento nei contesti discorsivi e scientifici più vasti (quello nazionale oppure quello patriarcale) sono temi che si possono seguire (e sono stati già seguiti) su vari esempi, tra cui i più emblematici sono sicuramente quelli del teatro napoletano e siciliano ${ }^{2}$. Mi riferisco agli esempi della tradizione teatrale meridionale (molto significativa ad articolata di per sé e piena anche di sfumature che meritano studi separati, ma che certamente non ricopre l'intero paesaggio del teatro dialettale italiano) soltanto per focalizzare l'attenzione di chi legge sulle due - ed

1 Cfr. per esempio Wilmer, 2004.

2 Cfr. per esempio i più recenti libri di: Angelini, 2002; De Miro D’Ajeta 2003; de Blasi, 2016; Madrignani, 2007; Barsotti, 2007 e 2009. 
a mio avviso - più importanti strategie di rappresentare la cultura locale che chiamerei qua: "l'etnonostalgia" e "l'etnoodio".

La prima consiste nel presentare la cultura locale come un mondo ormai perduto, appartenente al passato e prevede una drammaturgia in cui i personaggi vivono la propria realtà culturale come qualcosa che sta sparendo oppure estinguendosi a contatto con lo sviluppo economico e politico del paese, esattamente come i fratelli Saporito, apparecchiatori delle feste popolari de Le voci di dentro di Eduardo, che hanno dovuto chiudere la ditta del padre, dato che le feste del paese non si organizzavano più. Lo spettatore viene posizionato in questo caso nel ruolo del testimone del processo di sparizione di una cultura locale, verso la quale sviluppa un sentimento di nostalgia. Questa nostalgia oggi viene ancora rafforzata dall'onnipresenza del repertorio Eduardiano (e non solo Eduardiano per quanto riguarda il teatro dialettale) nel cinema, nella televisione ed in Internet sia al livello nazionale che globale. I media poi (soprattutto negli anni sessanta del Novecento) hanno diffuso e perfino pietrificato questa visione nostalgica della cultura meridionale italiana vista come metonimia di un'Italia plebea ed "ancestrale", come la pensava Pier Paolo Pasolini, ormai inesistente ed appartenente al passato ${ }^{3}$. Questo tipo di proiezione mediatica della cultura locale, come diceva a sua volta Arjun Appadurai - il quale ha analizzato la circolazione nel contesto globale delle cosiddette "etnoimmagini”" nell'epoca della televisione ed Inter-

3 Vedi a proposito Taviani, 1997, p. 125 e anche il mio recente libro: Bal, 2017, pp. 235-269, in prossima uscita anche in inglese nella casa editrice Transcript Verlag. Quest'ultimo libro ripercorre anche la presenza mediatica del teatro dialettale nei tempi più recenti. Ovviamente, la visione eduardiana della cultura locale meridionale, come anche quella pasoliniana, è stata più volte ridiscussa nel contesto teatrale italiano, soprattutto da parte degli artisti del cosiddetto teatro di ricerca negli anni Ottanta del Novecento (per esempio da Leo de Berardinis, Annibale Ruccello, Enzo Moscato), per non parlare degli esempi cinematografici più recenti, tra cui spicca il film di Matteo Garrone Gomorra (2008) basato sull'omonimo romanzo di Roberto Saviano del 2006 e seguito poi da una serie televisiva dallo stesso titolo 2014-2019 prodotta da SkyItalia, Fandango, Cattleya, e LA7. Roberto Saviano insieme a Mario Geraldi è anche autore di un omonimo spettacolo teatrale basato sul romanzo, per il quale ha vinto gli Olimpici del Teatro 2008. 
net (Appadurai, 2005, p. 117) - costringe spesso lo spettatore a desiderare le cose che non ha mai avuto e non ha mai perduto, esattamente come avviene nella pubblicità moderna. Soddisfa anche il sempre più diffuso desiderio degli spettatori di contrastare gli aspetti negativi dello sviluppo economico globale e del processo di acculturazione delle società considerate nel quadro dell' etnografia e dell'antropologia novecentesca "arcaiche", "originali" o "rurali".

$\mathrm{Su}$ un lato opposto invece si posiziona, secondo me, la strategia drammaturgica che qui chiamerei "l'etnoodio". Si tratta di un percorso drammaturgico adottato da parte degli artisti dialettali per esprimere e rappresentare un rapporto conflittuale con il proprio ambiente culturale, rimettendo in discussione sia le dinamiche intrinseche ad una cultura locale (per esempio: il conflitto lingua-dialetto o centroperiferia, le relazioni fra uomo-donna) sia estrinseche (come il rapporto con 1'esterno: il resto dell'Italia oppure il resto del mondo). In questo contesto spicca senz'altro la proposta di lettura delle opere di Emma Dante intrapresa da Anna Barsotti (Barsotti, 2009), la quale posiziona la drammaturgia della regista palermitana nel contesto degli altri autori isolani colpiti dal cosiddetto "effetto Sicilia" oppure "sicilitudine"(Ibidem, pp. 9-39). La Barsotti interpreta i problemi sollevati dalla Dante (per esempio quelli di un difficile rapporto con il bagaglio culturale siciliano che affligge anche gli altri siciliani come Luigi Pirandello, Luigi Capuana, Pier Maria Rosso di San Secondo nella prospettiva antropologica e simbolica disegnata parzialmente già da Carlo Alberto Madrignani (2007). Vede quindi l'identità culturale siciliana dei suddetti autori come una qualità oggettivamente esistente, risultante dalla loro biografia, dall'appartenenza alla terra e alla comunità isolana. Si tratta però di un patrimonio culturale dal quale essi spesso fuggono (fisicamente o esteticamente) tentando di mettere in discussione la propria "sicilitudine". Pur concordando con la Barsotti su questa linea di pensiero e quindi cercando di approfondire il problema dell'identità culturale locale dalla prospettiva delle artiste femminili contemporanee come Emma Dante, vorrei comunque proporre qui un approccio un po' diverso da quello dalla studiosa italiana. 
Ispirandomi al concetto del "performativo" o "performático" (in inglese: performativity) sviluppato da Judith Butler (1993, 2004) - applicato da lei in riferimento al concetto del genere (Eadem, 2003) - vorrei considerare l'identità culturale locale come una pratica insieme discorsiva e corporale che si deposita nei corpi delle persone e che, a forza di continue ripetizioni, crea un effetto naturalizzante. Va quindi vista non come una caratteristica essenziale, ma piuttosto come un atto performativo e ripetitivo, il quale nello stesso tempo ha una forza oppressiva nei confronti delle comunità stesse ed in particolare nei confronti delle donne. A mio avviso la cultura locale siciliana, più volte ridiscussa dalla Dante, diventa una finzione regolativa, la quale serve piuttosto per mantenere uno status quo, in cui gli uomini esercitano un potere dominante e discriminatorio nei confronti delle donne. Vorrei quindi analizzare due esempi degli inizi di carriera dell'artista siciliana: mPalermu del 2001 (Premio Ubu 2002) e Carnezzeria del 2002 (Premio Ubu 2003), i quali, come una lente di ingrandimento, costituiscono uno strumento epistemologico per capire meglio il funzionamento della realtà culturale locale nel contesto del mondo globalizzato odierno. Questo aspetto mi sembra poi particolarmente importante da approfondire, essendo io stessa traduttrice polacca delle opere teatrali di Emma Dante ${ }^{5}$, che segue il suo lavoro da una certa distanza ${ }^{6}$.

Vorrei comunque iniziare la mia analisi partendo da un aneddoto che la stessa Dante ha raccontato mentre stava lavorando ad un altro spettacolo, intitolato Cani di bancata (dedicato alla mafia siciliana, del 2006), un aneddoto che, secondo me, illustra molto bene il suo rapporto dialettico con la cultura locale:

4 Senza aprire un dibattito lungo sulla provenienza di questo termine nel contesto scientifico italiano rimando al libro di Fabrizio Deriu (2012) e al suo recente articolo (2015, pp. 193-208) e a Marco de Marinis (2008).

5 Cfr. Dante, 2011 e Eadem, Le Pulle, traduzione dello spettacolo per il Festival Internazionale di Teatro "Dialog" Wrocław 2011. Vedi anche le mie traduzioni degli autori italiani che scrivono in dialetto: Davide Enia, Enzo Moscato, Annibale Ruccello in Bal, 2007.

6 Ho visto i suoi spettacoli soprattutto durante le tournée europee, dove i codici della cultura dialettale italiana non sempre vengono captati dal pubblico internazionale, contano invece ben altri aspetti della sua opera, come la ripetizione gestuale e l'ermeticità discorsiva. 
In Sicilia abita un popolo che parla un gergo segreto, accompagnato da ammiccamenti, da gesti con le mani, la testa, gli occhi, le spalle, la pancia, i piedi. Un popolo capace di fare tutto un discorso senza mai aprire bocca. Questo popolo ha un atteggiamento mafioso che non ha niente a che vedere con la mafia. Faccio un esempio: sto percorrendo in auto una stradina a senso unico e di fronte a me arriva un'auto contromano. Mi fermo, ho fretta e suono il clacson. Aspetto che il conducente indietreggi e, nonostante il mio coraggio, basta un suo sguardo accompagnato da un cenno colla testa per farmi capire che mi conviene fare retromarcia. (http://www.emmadante. com/canidibancata.html)

In Sicilia, secondo la Dante, la omertà insieme alla cerimoniosità dei comportamenti quotidiani compongono una specie di codice "mafioso", improntato in certi gesti e nei modi di dire ormai automatizzati che trasformano le persone nei soggetti ubbidienti alla forza oppressiva della tradizione. Questa tradizione però non è affatto sinonimo del patrimonio culturale, etnograficamente e antropologicamente inteso, ma piuttosto un atto di sottomissione dei suoi personaggi alle pratiche culturali locali, quali l'uso del dialetto, dei modi di dire, dei gesti quotidiani e dei cenni con la testa. La sua trilogia dedicata alla famiglia palermitana composta da spettacoli mPalermu (2001), Carnezzeria (2002) e Vita mia (2004) costituisce a mio avviso il miglior esempio dell'analisi critica di questa sottomissione (Dante, 2007).

Quando si pensa alla famiglia italiana rappresentata nel teatro dialettale del Novecento inevitabilmente viene in mente la drammaturgia di Eduardo, il quale quasi sempre ritraeva la famiglia popolare o piccolo borghese sullo sfondo di un basso o di una casa condominiale, famiglia la cui integrità veniva spesso minacciata dal processo di sviluppo civilizzazionale del paese. La visione di Eduardo fa ovviamente parte di un lungo filone della drammaturgia dialettale che, almeno a partire dalla metà dell'Ottocento, ha deriso e straniato i modelli familiari proposti dal teatro borghese ${ }^{7}$ e ha messo in rilievo i tortuosi aspetti dell'unificazione dell'Italia. Quello che mi sembra più importante da notare in questo mo-

7 Basta pensare al filone: Antonio Petito, Eduardo Scarpetta, Eduardo de Filippo da una parte e dall'altra al neogoldonismo, a Giuseppe Giacosa e a Luigi Pirandello (Angelini, 1997, pp. 34-46). 
mento è che la famiglia napoletana di Eduardo, essendo ormai entrata nell'immaginario italiano come sinonimo di una famiglia popolare italiana di per sé, ha costituito anche un accattivante e avvolgente modello drammaturgico della cultura locale. Un modello decisamente da abbandonare per la Dante, indipendente dall'uso del dialetto in scena.

Il suo uso del dialetto in scena (e delle altre lingue e dialetti, come il francese e il napoletano) non sembra però affatto descrittivo e deittico, cioè non rinvia direttamente ad una realtà culturale effettivamente esistente (almeno per il pubblico internazionale). I personaggi in scena parlano siciliano, ma questo parlare è quasi irriconoscibile come dialetto, in quanto gli attori spesso sussurrano le parole, le ripetono velocemente, quasi apposta per non essere compresi. Parlano una lingua che nasconde i sensi e costringe lo spettatore a dedurre i significati anziché comprenderli. Quindi il dialetto rimane per la Dante un linguaggio segreto, ermetico, appunto un codice di comportamento misterioso che lascia gli spettatori di fronte ad un'impenetrabilità dei modi di fare siciliani. Oltre alla lingua e alla gesticolazione corporea, la Dante non si serve poi di quasi nessun altro attrezzo scenografico che possa essere facilmente ricondotto al contesto culturale siciliano: nessun tavolo, nessuna stanza, nessun basso.

I personaggi di Emma Dante non si avvalgono di paesaggi, di "etnoimmagini" o semplicemente dei luoghi comuni della cultura dialettale. I membri della famiglia Carollo, protagonisti de mPalermu - Nonna Citta, Zia Lucia, Rosalia, Mimmo e Gianmarco - sono stati semplicemente allineati lungo la luce della ribalta, come se, tra poco, dovessero attraversare la quarta parete o varcare la soglia del palcoscenico per raggiungere gli spettatori, quasi nel gesto di superare i propri vincoli culturali. Si esortano a vicenda ad uscire, dicendo «Mimmo: Niscèmu?, Giammarco: Niscèmu!» (Dante, 2007, p. 50). Ogni volta, però, qualche sciocchezza, qualche futile ostacolo, gli impedisce di uscire, di eseguire quel passo apparentemente facile verso il mondo, al di fuori dal proprio contesto culturale: Rosalia non ha fatto in tempo a mettere le scarpe da visita ed è rimasta in ciabatte, Mimmo indossa i pantaloni troppo corti e quando qualcuno nota questo fatto imbarazzante va su tutte le furie, cominciando a flagellare i familiari intorno a sé. Uscire dal tea- 
tro, dal palcoscenico, dalla cornice teatrale equivale, nello spettacolo di Emma Dante, all'uscita dalla propria appartenenza culturale, ossia dall'identificazione culturale dei palermitani. Purtroppo ogni volta quel passo decisivo deve essere compiuto, i personaggi si tirano indietro, eseguendo gesti e intraprendendo azioni che li allontanano dal traguardo. Cominciano per esempio a raccontare un viaggio, a correre dietro ad un pallone, si strappano dalle mani una busta con i dolci. Finalmente, all'improvviso, arriva la pioggia che bagna tutti sul palco, e sembra per un attimo, che tutta questa famiglia rumorosa e fin troppo distratta si ricomponga e ricominci ad agire secondo lo scenario prestabilito. Infatti, la zia Lucia incoraggia ancora una volta tutti ad uscire, dicendo «Usciamo in italiano!» (Ibidem, p. 64), come se uscire in siciliano fosse stato troppo difficile finora. All'ultimo momento purtroppo Nonna Citta comincia a piegarsi dal dolore per aver mangiato troppi dolci e tutta la famiglia, ancora una volta, fa un passo indietro.

In questo spettacolo, uno dei suoi primi, la Dante ha voluto dimostrate molto chiaramente come i gesti, le parole e gli sguardi quotidiani, apparentemente banali e superficiali (riprodotti freneticamente dagli attori sul palco), costituiscono un grave ostacolo nel processo di integrazione culturale della comunità siciliana nel contesto globale. Per illustrare questa difficoltà la Dante ha deciso di usare la corporeità dei propri attori, costringendoli a costruire i personaggi scenici tramite una serie di gesti, quasi tick ossessivi, fiumi di parole e ritornelli vocali. Questo metodo dimostra molto bene quanto l'identità culturale in generale sia in fondo puramente performativa, essendo frutto di un'iterazione di gesti e di parole che non sono affatto "naturali", ma sembrano tali a forza delle continue ripetizioni. Il fatto che un'identità, la si possa imparare o recitare sembra abbastanza inquietante, specialmente poi quando essa serve a camuffare l'aggressione e la violenza nei rapporti tra uomo e donna. Il termine "siciliano", associato ingenuamente ai gesti e ai costumi locali o "etnici", troppo spesso, secondo la Dante, nasconde poi la violenza di una famiglia patriarcale, dove la "tradizione" serve piuttosto come scusa per abusi sessuali.

Per svelare queste relazioni familiari perverse la Dante ha prodotto lo spettacolo Carnezzeria, al centro del quale vediamo la protagonista 
Nina, vestita nell'abito da sposa e incinta, che aspetta il suo sposo sul palcoscenico. Lo sposo deve prenderla in moglie per salvarla dall'odio sociale. Dal comportamento della ragazza deduciamo il suo ritardo mentale e osserviamo come la donna obbedisce passivamente, senza riflettere, alla volontà della famiglia. Nel tempo di attesa della cerimonia i tre fratelli della ragazza si divertono a fare scherzetti e gesti sconci, quasi come se stessero gareggiando nel concorso di virilità. Ad un certo momento, cominciano un dibattito che si trasforma improvvisamente in una lite sul contenuto di una fotografia che prima solo la ragazza e poi loro tre tengono in mano. Tra una battuta e l'altra dei maschi, gli spettatori scoprono che le persone ritratte nella foto (i membri della famiglia) sono stati a lungo abusati sessualmente dal padre, così come Nina stessa è stata abusata anche dai tre fratelli. Tra le battute pesanti e le risate dei tre uomini, sembra impossibile riparare o per lo meno analizzare queste relazioni familiari violente.

Nina soprattutto non ha nessuna voce in capitolo. Non protesta neanche quando i fratelli le danno calci nella pancia oppure quando cercano di soffocarla schiacciandola sul collo con i piedi. Finalmente, nell'ultima scena, Nina - quasi eseguendo un ordine della famiglia - muore strozzata dal suo velo nuziale. Obbedisce all'ordine di pulire la famiglia dal peccato dell'incesto, ostentato dalla sua gravidanza, e muore nella scena finale. Ma è uno strozzamento, un'impiccagione molto particolare. Perché il suo velo nuziale non viene attaccato in alto, ma proprio in basso, sigillato al suolo. Nina muore quindi impiccata, ma paradossalmente piegata al proprio territorio. Una scena simbolica, quindi, nella quale la Dante attribuisce la colpa della morte della ragazza a quello che il territorio rappresenta: l'apparato gestuale e discorsivo oppressivo che nasconde le atrocità sessuali.

Con questo spettacolo la Dante ha provocato un'intensa discussione in Italia, svelando i meccanismi grazie ai quali certi crimini che avvengono nelle comunità locali rimangono nascosti o taciuti. Franco Quadri ha scritto ne "La Repubblica" (21.11.2002): «è un capolavoro costruito con ritegno essenziale che esplode come una bomba grazie ad un silenzio che denuncia (...) lascia ammaliati ed esterrefatti, senza respiro ne parole». Mentre Renato Palazzi ha sottolineato «la rivisitazione cerimo- 
niale delle foto di famiglia è il pretesto per evocare le insicurezze, le aggressività e le ossessioni maschiliste degli uomini e la subalternità della donna, eterni emblemi di una terra che cambia i modi di rappresentarsi ma non i fondamenti del proprio pensiero ancestrale» (Palazzi, 2002). Questa analisi sembra forse ancora più pungente se pensiamo che la "sicilianità" o qualsiasi altra caratteristica della cultura locale in Europa riguarda ormai le comunità culturalmente avanzate. Disfarsi quindi della propria circoscrizione culturale, della cosiddetta "natura ancestrale" o primordiale per guardarla con occhio più critico e distaccato acquisisce oggi un'estrema importanza, visto che per la Dante l'identità culturale locale non è affatto una qualità da salvare e nemmeno l'oggetto di un nostalgico ricordo, ma piuttosto un problema sociale e politico che merita un dibattito pubblico.

In questo senso i suoi spettacoli permettono di guardare sotto una luce molto più critica il concetto di etnoimmagini proposto da Arjun Appadurai, secondo il quale la nozione del locale potrebbe essere ricondotta ad una drammatizzata visione nostalgica di una comunità rurale, alla curiosità $\mathrm{o}$ all'attrazione turistica nel mondo sempre più globalizzato e mediatizzato. Il problema di come rappresentare la cultura locale va indagato invece dal punto di vista delle pratiche decoloniali, le quali smascherano il morboso e sempre insoddisfatto desiderio del mondo globalizzato di conoscere l'Altro per ripristinare i vecchi concetti di sudditanza delle donne agli uomini nella nuova veste dell'esotismo. Si tratta di uno sguardo del tutto patriarcale e padroneggiante che viene soddisfatto con le varie rappresentazioni delle "donne dell'Islam" o "della prostituzione infantile" in Tailandia - come spesso esclamano le testate dei tabloid. In quest'ottica l'aggettivo "siciliano", come quello "arabo" o "orientale", stimola l'immaginazione del pubblico occidentale affamato dell'esotismo. Ho l'impressione, però, che Emma Dante, anziché lottare con questo sguardo neocoloniale e voyeristico, non lo disdegni affatto. Anzi, forse per lei si tratta di una curiosità salvifica, perché comunque aiuta a denunciare le politiche oppressive che stanno dietro le cosiddette identità culturali locali. La Dante mette quindi lo spettatore a confronto con la parte più oscura delle pratiche "culturali" della propria comunità forse per ricondurlo ad una presa di coscienza. Tutto 
sommato lo spettatore delle opere di Emma Dante non sta assistendo ad un documentario di National Geographic, in cui i leoni mangiano le gazzelle in piena serenità, mentre la voce suadente del giornalista spiega la drammaturgia dell'accaduto. Non si tratta di guardare le etnoimmagini di questo tipo. I problemi trattati dalla Dante vengono piuttosto trasmessi attraverso una lotta corporale degli attori con la propria identità culturale, vissuta come un trauma. Lo vediamo soprattutto grazie all'abilità e alla sveltezza degli attori, al loro contatto corporale reciproco che sfiora la violenza. Quindi possiamo dire che lo spettatore del teatro di Emma Dante viene messo in una posizione in cui continuare a guardare quello che succede sul palcoscenico diventa problematico dal punto di vista etico. Mettendo lo spettatore a confronto con la violenza la Dante pretende dal pubblico una reazione: resta o scappa, oppure fai qualcosa. Lo mette quindi nei panni di quell'autista dell'aneddoto raccontato all'inizio il quale, pur guidando nel senso giusto, deve decidere se vuole cedere di fronte all'avversario arrogante oppure affrontarlo. La Dante ripristina quindi al teatro quella funzione che avevano le performance di Marina Abramovic negli anni 60 del XX secolo (molti di loro indagavano proprio i rapporti violenti tra uomo e donna). Una funzione che va studiata secondo le modalità proposte da Erika Fischer-Lichte in Estetica del performativo (2014). Si tratta quindi di indagare il rapporto tra gli spettatori e gli attori che si instaura grazie al reciproco scambio di energie e di feedback autopoietico. Le arti performative costituiscono infatti un veicolo che reindirizza la percezione dello spettatore dal campo di contemplazione puramente estetica al campo di riflessione etica. E proprio in questo passaggio consiste, secondo me, la forza del teatro di Emma Dante e il suo potere di cambiare la situazione delle donne attraverso un atteggiamento critico nei confronti della cosiddetta cultura locale.

\section{BIBLIOGRAFIA}

Angelini, F. (2003). Rasoi: I teatri napoletani del 900. Roma: Bulzoni. Appadurai, A. (1996). Modernity at Large. Cultural Dimensions of Globalisation. Minneapolis, London: University of Minnesota Press. 
Appadurai, A. (2005). Nowoczesność bez granic. Kulturowe wymiary globalizacji. Kraków: Universitas.

Bal, E. (2017). Lokalność i mobilność kulturowa teatru. Kraków: Wydawnictwo Uniwersytetu Jagiellońskiego.

Bal, E. (2011). Ceremonie zamiast słów. In E. Dante, Trylogia o okularach (pp. 7-19). Kraków: Pangapak.

Bal, E. (2007). W podróży po językowym krajobrazie Włoch. In E. Bal (Ed.), Na jeden i kilka głosów. Sztuki włoskie (pp. 7-27). Kraków: Pangapak.

Barsotti, A. (2007). Eduardo-Fo e l'attore-autore del Novecento. Roma: Bulzoni.

Barsotti, A. (2009). La lingua teatrale di Emma Dante, "mPalermu”, "Carnezzeria", "Vita mia". Pisa: ETS.

Butler, J. (2002). Antigone's Claim. Kinship Between Life and Death. Irvine: Columbia University Press.

Butler, J. (1993). Bodies that Matter. On the Discoursive Limits of Sex. New York, London: Routledge.

Butler, J. (2004). Undoing Gender. New York, London: Routledge.

Carlson, M. (2001). The Haunted Stage. The Theatre as Memory Machine. Ann Arbor: University of Michigan Press.

Carlson, M. (2006). Speaking in Tongues, Language at Play in the Theatre. Ann Arbor: The University of Michigan Press.

Clifford, J. (1997). Routes. Travel and Translation in the Late Twentieth Century. Harvard: Harvard University Press.

Dante, E. (2007). Carnezzeria. Trilogia della famiglia siciliana. Roma: Fazi.

De Blasi, N. (2016). Eduardo. Salerno: Salerno.

De Marinis, M. (2008). Introduzione alla presente edizione. Teoria, pratica e storia: problemi metodologici degli studi teatrali. In: M. De Marinis, Capire il teatro. Lineamenti di una nuova teatrologia. Seconda edizione rivista ed ampliata (pp. 13-30). Roma: Bulzoni.

De Miro D’Ajeta, B. (2003). La figura della donna nel teatro di Eduardo del Filippo. Napoli: Liguori.

Deriu, F. (2012). Performático. Teoria delle arti dinamiche. Roma: Bulzoni.

Deriu, F. (2015). La ricezione dei Performance Studies in Italia. Appunti di cronaca e riflessioni epistemologiche. In G. Guccini \& G. Petrini (Eds.), "Thinking the Theatre. New Theatrology and Performance Studies". Atti del Convegno CUT (pp. 193-208). Bologna: University Digital Library. 
Fischer-Lichte, E. (2008). Estetyka performatywności. Kraków: Księgarnia Akademicka.

Fischer-Lichte, E. (2014). Estetica del performativo. Una Teoria del teatro e dell'arte. Roma: Carocci.

Madrignani, C.A. (2007). Effetto Sicilia. Genesi del romanzo moderno (Verga Capuana De Roberto Pirandello Tomasi di Lampedusa Sciascia Consolo Camilleri). Macerata: Quodlibet.

Palazzi, R. (2002). Una macabra festa di nozze siciliana. Retrieved from http:// www.emmadante.com/wp-content/uploads/Carnezzeria4.pdf.

Taviani, F. (1997). Uomini di scena, uomini di libro. Introduzione alla letteratura teatrale italiana del Novecento. Bologna: il Mulino.

Quadri, F. (2002, November 21). Festa di nozze con cadavere. la Repubblica, 271. Retrieved from http://www.emmadante.com/wp-content/uploads/ Carnezzeria1.pdf.

Wilmer, S.E. (Ed.). (2004). Writing and Rewriting National Theatre Histories. Studies in Theatre History and Culture. Iowa City: University of Iowa Press.

\section{Spettacoli analizzati:}

mPalermu, testo e regia di Emma Dante, prima rappresentazione al Teatro del Parco a Parma nel novembre 2001.

Carnezzeria, testo e regia di Emma Dante, prima rappresentazione al Teatro dell'Arte a Milano nel 2002.

Riassunto: Prendendo in considerazione i cambiamenti avvenuti negli ultimi 20 anni nel campo degli studi sulla performance e sulle rappresentazioni delle culture locali ed etniche e le metodologie elaborate nel campo degli studi de- e post -coloniali, Ewa Bal propone di rivedere le principali strategie di rappresentazione delle culture locali nel teatro dialettale italiano dell'ultimo decennio. Parte dunque dal presupposto che il modello drammaturgico elaborato da Eduardo de Filippo sia rimasto a lungo egemonico nel contesto teatrale italiano: specie nella maniera in cui produceva la nostalgia di un passato idealizzato della cultura dialettale. Questo modello, secondo l'autrice, va invece rivisto mettendo al suo confronto le artiste più recenti del teatro dialettale, che concettualizzano la cultura locale in maniera differente e propongono nuove strategie drammaturgiche. Bal analizza quindi le strategie performative di due opere di Emma Dante mPalermu e Carnezzeria per dimostrare che la cosiddetta identità culturale locale non è affatto una caratteristica essenziale (o "naturale") di una certa comunità, ma piuttosto un insieme di pratiche discorsivo-corporali ripetibili che a forza di ripetersi diventano "naturali". Vanno quindi viste piuttosto come una specie di minaccia o stigma culturale, che serve da camuffaggio alle più profonde relazioni di dominazione e violenza tra uomini e donne. Analizzando due esempi di spettacoli di Emma Dante risalenti agli inizi della sua carriera artistica, Bal guarda criticamente alla nozione del "primordialismo" sollevata da Arjun Appadurai 
e interpretata da lui come uno dei modi di rappresentare l'Altro che soddisfa lo sguardo patriarcale del mondo Occidentale nei confronti delle culture apparentemente meno avanzate o primordiali. Gli spettacoli di Emma Dante sovvertono questo sguardo voyeuristico, usandolo criticamente per denunciare la violenza nascosta sotto gli scenari comportamentali, gestionali e discorsivi della cultura locale siciliana e riportarla alla luce del dibattito pubblico.

Parole chiave: località, identità culturale, teatro dialettale, Emma Dante, performativo 